\title{
Consideraciones éticas sobre el uso de metotrexate en el embarazo tubario (ET)
}

\author{
María de la Luz Casas M. ${ }^{1}$, Rosa María Sánchez Javier ${ }^{2}$
}

Resumen: El embarazo tubario (ET) es la implantación de un óvulo fertilizado en la trompa uterina. El hecho es que el ET seguirá presentándose muchas veces al médico en forma de un episodio clínico urgente de hemorragia intraabdominal. El médico puede dar entonces por segura la muerte del embrión e intervendrá para extirpar los restos embrionarios y la trompa perforada y sangrante. Conseguirá así revertir la grave amenaza inmediata a la vida de la madre. El tratamiento médico con metotrexate (MTX) es un procedimiento seguro y efectivo en el tratamiento del embarazo ectópico, sin los riesgos potenciales de la cirugía. La acción del médico no será atentar directamente sobre el embrión, sino sobre la causa de la patología, que quizás provoque la muerte del embrión. La intervención medicamentosa en estos casos ha sido considerada por algunos como una forma de aborto indirecto y, por tanto, en apreciación de la invulnerabilidad de la vida humana, un acto antiético. Pero la argumentación ética sobre el objetivo de esta intervención puede ser postulada bajo el principio del doble efecto.

Palabras clave: embarazo tubario, metotrexate, embrión, embarazo de alto riesgo, bioética

\section{Ethical considerations about the use of metotrexate in ectopic pregnancy (TP)}

\begin{abstract}
Ectopic pregnancy (EP) consists in the implantation of a fertilized ovule in the fallopian tube. The fact is that EP will continue to appear many times to physicians in the form of an urgent clinical episode of intra abdominal bleeding. The physician may consider then as certain the death of the embryo and he/she will intervene to extirpate the embryo remains and the bleeding and perforated fallopian tube. In this way he/she will revert the serious immediate threat to the life of the mother. The medical treatment with metotrexate (MTX) is a safe and effective procedure in the treatment of ectopic pregnancy, without the potential risks of surgery. The act of the physician will never be to attempt directly against the embryo, but on the cause of the pathology, that may provoke the death of the embryo. The medical intervention in these cases has been considered by some as a type of indirect abortion and, therefore, an act contrary to ethics, considering the invulnerability of human life. But the ethical argumentation about the objective of this intervention may be posed under the principle of double effect.
\end{abstract}

Key Words: ectopic pregnancy, metotrexate, embryo, high risk pregnancy, bioethics

\section{Consideraciones éticas sobre o uso de metotrexate na gravidez tubária (GT)}

Resumo: A gravidez tubária (GT) é a implantação de um óvulo fertilizado na trompa uterina. O fato é que a GT seguirá apresentando-se muitas vezes ao médico em forma de um episódio clínico urgente de hemorragia intra-abdominal. O médico pode dar entâo por seguro a morte do embriáo e intervirá para extirpar os restos embrionários e a trompa perfurada e sangrante. Conseguirá assim reverter a grave ameaça imediata à vida da mãe. O tratamento médico com metotrexate (MTX) é um procedimento seguro e efetivo no tratamento da gravidez ectópica, sem os riscos potenciais da cirurgia. A ação do médico não será nunca atentar diretamente contra o embrião, senão sobre a causa da patologia, que quiçá provoque a morte do embrião. A intervenção medicamentosa nestes casos tem sido considerada por alguns como uma forma de aborto indireto e, portanto, em apreciação da invulnerabilidade da vida humana, um ato antiético. Porém, a argumentaçáo ética sobre o objetivo desta intervenção pode ser postulada sob o princípio do duplo efeito.

Palavras-chave: gravidez tubária, metotrexate, embrião, gravidez de alto risco, bioética

\footnotetext{
${ }^{1}$ Doctor en Ciencias, Bioética. Jefe del Departamento de la Escuela de Medicina de la Universidad Panamericana, México Correspondencia: mcasas@up.edu.mx

${ }^{2}$ Médico Gíneco-Obstetra. Profesor de la Escuela de Enfermería de la Universidad Panamericana, México
} 


\section{Aspectos médicos del embarazo tubario}

El embarazo ectópico es la implantación de un óvulo fertilizado fuera de la cavidad endometrial(1). Se produce con más frecuencia en la trompa de Falopio, pero puede también ocurrir en el ovario, entre el ligamento ancho, dentro del canal cervical o cualquier otro lugar de la cavidad abdominal. El denominador común de muchas teorías acerca de la causa es la demora en el transporte del óvulo; normalmente hay un factor que altera el transporte del óvulo, como es la lesión de la superficie de la mucosa ciliada, originada por infección o inflamación. Otra de las teorías menciona un defecto en el embrión, pero no se ha demostrado incidencia mayor de anomalías cromosómicas en los embarazos ectópicos que en los abortos. Las alteraciones hormonales también se han considerado como factor etiológico, por los efectos que pueden causar los estrógenos y progesterona sobre la actividad del músculo liso y ésta, a su vez, sobre la movilización del óvulo fecundado; también se ha observado una incidencia mayor en la raza negra que en la blanca.

Dentro de las causas anatómicas se pueden mencionar: enfermedad pélvica inflamatoria, salpingitis ocasionada por el aumento en las enfermedades de transmisión sexual y el aumento es mayor después de una salpingitis tuberculosa, en aproximadamente $35 \%(2)$. Otro factor puede ser también la existencia de cirugías previas, realizadas en forma primaria como cirugías reconstructivas por esterilidad, que pueden ocasionar estenosis intraluminal tubaria. La tasa de embarazo ectópico después de falla de esterilización tubaria varía entre el 15 y $75 \%$, según la técnica utilizada, siendo la esterilización con coagulación monopolar la que da la cifra más alta de embarazos ectópicos posteriores. Los abortos inducidos aumentan diez veces la incidencia de embarazos ectópicos, probablemente por infecciones no detectadas asociadas al procedimiento. Los dispositivos intrauterinos (DIU) no aumentan la incidencia absoluta de embarazos ectópicos, pero si una mujer se embaraza con DIU aumenta la incidencia relativa de embarazos tubarios; en estos casos la posibilidad es de 1 por cada 23 embarazos(3). El tipo de DIU más frecuentemente asociado a embarazo ectópico es el que contiene progesterona; la causa no se ha definido, pero se plantea que el efecto del DIU en la motilidad tubaria desacelera el transporte del óvulo.

Las anomalías del desarrollo de la trompa, como los divertículos, las aberturas accesorias y la hipoplasia, aumentan la incidencia de embarazo ectópico. Los factores que retardan el paso del óvulo fecundado por el oviducto son: emigración externa del óvulo, reflujo menstrual, motilidad tubaria alterada por medicamentos hormonales - como estrógenos o progestágenos-, medicamentos que aumentan la receptividad del ovulo, hormonas o endometriosis. Otro factor es la migración de un óvulo desde un ovario a la trompa contralateral: el retraso en el transporte del huevo puede favorecer la localización ectópica de la implantación(4). La inseminación intrauterina, la fertilización in vitro y el GIFT aumentan la incidencia del embarazos ectópicos. El uso de fármacos estimulantes de la ovulación, como la gonadotropina coriónica humana y citrato de clomifeno, predispone a la implantación extrauterina. También se han informado anormalidades del cigoto en el embarazo ectópico, incluyendo anomalías cromosómicas, malformaciones francas y defectos del conducto neural, y también un aumento de la frecuencia de embarazo ectópico en compañeras de varones que tienen cuentas anormales de espermatozoides o frecuencia elevada de espermatozoides defectuo$\operatorname{sos}(5)$.

El tratamiento depende del lugar y del tamaño de embarazo, el estado de la trompa y los deseos de la paciente por conservar la fertilidad. Puede ser médico o quirúrgico; si es quirúrgico puede ser por laparotomía o laparoscopía; algunos embarazos tubáricos serán reabsorbidos espontáneamente, pero un 10 a $30 \%$ de ellos requerirá un tratamiento quirúrgico posterior(6). Para efectos de este artículo nos referiremos solamente al embarazo tubario (ET).

El diagnóstico normalmente es la presencia de un dolor abdominal o pélvico y el sangrado vaginal, que son las manifestaciones más importantes en el primer trimestre. Los síntomas pueden ser erráticos, variables y en algunos casos ausentes. En la exploración física, la hipotensión, taquicardia, defensa abdominal y rebote, acompañado de dolor de anexos, aun sin tumoración, reflejan un pronóstico grave por amenaza de ruptura, requirien- 
do de atención quirúrgica inmediata. La mayoría de las pacientes se presentan con un cuadro menos crítico, lo que hace posible que se pueda ofrecer un tratamiento médico, evitando una cirugía.

La laparotomía continúa siendo el tratamiento de elección en la paciente hemodinámicamente inestable, con posibilidad de ruptura uterina.

El tratamiento médico con metotrexate (MTX) (misoprostol) es un procedimiento seguro y efectivo en el tratamiento del embarazo ectópico, sin los riesgos potenciales de la cirugía. Fue introducido en 1982 por Tanaka, cuando lo utilizó por primera vez para un caso de gestación intersticial. Su eficacia es similar a la de la salpingostomia laparoscópica, oscilando el porcentaje de pacientes tratadas satisfactoriamente entre 80 y $100 \%$, con una media de 95\%. Existen diferentes técnicas de aplicación del MTX: vía sistémica, mediante dosis repetidas o mediante una sola dosis, y la vía local mediante inyección directa(7). Puede administrarse por vía oral, intramuscular, local, intratecal o por infusión continua. La inyección intramuscular es la vía preferida. El MTX puede usarse como tratamiento primario, tratamiento de ectópico persistente después de salpingectomía, profilaxis por sospecha de persistencia de productos de la concepción, después de tratamiento conservador quirúrgico y en casos de inusuales de ectópico. El folinato cálcico (leucovorin), uno de varios derivados activos, químicamente reducidos del ácido fólico, es útil como antídoto del MTX, lo cual permite dosis más altas de MTX al prevenir algunos de sus efectos secundarios y conservar células normales y prevenir toxicidad celular $(8,9)$.

En el esquema de multidosis, el MTX se administra $1 \mathrm{mg} / \mathrm{Kg} /$ dia intramuscular los días 1,3,5,7, y leucovorin como sal de calcio los días 2, 4, 6 y 8 $(0,1 \mathrm{mg} / \mathrm{Kg}$ intramuscular). Se puede administrar un segundo tratamiento si existe aumento o meseta en dos valores consecutivos de dosificación de fracción B de GCH (gonadotropina coriónica).

El esquema de dosis única simplifica la administración. La dosis única utiliza $50 \mathrm{mg} / \mathrm{m} 2$, vía inyección intramuscular, y no utiliza leucovorin. Con este esquema, la dosis se puede repetir después de una semana, si la dosificación de fracción $\mathrm{B}$ de $\mathrm{GCH}$ no disminuye por lo menos 15\% en- tre el día 4 y 7 después del tratamiento. Utilizando este esquema de dosis única, un $20 \%$ de las pacientes requieren más de un ciclo de tratamiento.

Para evitar negligencia con los esquemas de MTX, las pacientes deben monitorizarse semanalmente después del tratamiento, con dosificación de fracción B de GCH hasta que no se detecte (valor 0). Se debe tener en cuenta que los embarazos ectópicos pueden causar ruptura tubaria aun cuando los niveles de gonadotropinas se encuentren en fase descendente, y también esto se puede presentar en los casos en los que después de 48 horas del tratamiento con MTX la dosificación de fracción B de GCH aumenta por arriba del 66\%. En casos en los que, después de la administración de MTX, las gonadotropinas continúan aumentando, la ruptura puede ser tan alta como de $20 \%$.

La efectividad del MTX fue revisada por Pisarka y cols. basados en 12 estudios con 20 pacientes cada uno, y concluyeron que el tratamiento con MTX es efectivo en el 78 a $96 \%$ de las pacientes; el $78 \%$ mostró permeabilidad tubaria postratamiento, el $65 \%$ que intentó embarazo subsecuente tuvo éxito y el 13\% repitió el ectópico. El MTX ha sido usado en localizaciones cervicales, intersticiales, ováricas y abdominales, con resultados de hasta 80 a 90\% de éxito.

Antes de administrar MTX se debe pedir a la paciente un examen completo de sangre, pruebas de funcionamiento hepático y panel de electrolitos, creatinina sérica, exámenes de gabinete y placas de tórax, con el objeto de descartar riesgo de neumonitis intersticial.

Existen indicaciones, contraindicación y efectos secundarios en la utilización de este fármaco, datos que se omitirán por tratarse de un análisis ético de su uso y no uno de revisión ginecológica. Existen otros manejos de esta patología pero, por igual objetivo, no serán tratados en este documento.

El hecho es que el ET seguirá presentándose muchas veces al médico en forma de un episodio clínico urgente de hemorragia intraabdominal. El médico puede dar entonces por segura la muerte del embrión e intervendrá para extirpar los restos embrionarios y la trompa perforada y sangrante. 
Conseguirá así revertir la grave amenaza inmediata a la vida de la madre. Sobre este punto existe un acuerdo prácticamente universal entre médicos y moralistas.

Los procedimientos para el diagnóstico del ET se han ido perfeccionando considerablemente en años recientes y se ha hecho posible, mediante la determinación cuantitativa seriada de la betahCG sérica y la ecografía transvaginal de alta resolución, el diagnóstico precoz del ET antes de que éste haya dado síntomas clínicos o señales de inminente ruptura. Permiten también comprender cada vez mejor las posibilidades evolutivas del ET y su variado curso clínico, determinar el estado de viabilidad del embrión y diagnosticar su muerte, y una mejor individualización del tratamiento que debe administrarse a cada paciente. Es por este motivo, pues existe elección de manejo, que se presenta el dilema ético.

\section{Aspectos éticos}

Una de las máximas de la profesión médica señala que todo acto médico debe realizarse con calidad científica y ética. La ética también considera que las circunstancias son un factor importante que modifica las acciones éticas y no solamente la intención y el fin. El embarazo tubario constituye una patología gineco-obstétrica y, por tanto, los actos deben tener como objetivo el otorgar tratamiento que conserve la beneficencia y prevenga la maleficencia.

Ante un embarazo, el médico tiene dos pacientes a su cargo y en ninguna forma debe menospreciar la vida del embrión, el cual constituye un ser humano en desarrollo(10).

Sabemos que la bioética está fundamentada sobre cuatro principios básicos(11):

No maleficencia, que establece que el médico debe causar el menor prejuicio a su paciente. No provocar daños, ni agravios a la salud del paciente. Este principio se basa en primun non nocere del aforismo hipocrático.

Justicia, que establece la equidad como condición esencial del médico y determina su imparcialidad impidiendo actos discriminatorios que interfieran la buena relación médico paciente.
Beneficencia, que establece la búsqueda del bien mejor. Determina que la acción médica debe ser usada con sentimientos de filantropía y de amor por el ser humano.

Autonomía, que determina que las personas tienen derecho a decidir sobre cuestiones relacionadas con su cuerpo y con su vida. La conducta médica debe ser, previo conocimiento, autorizada por el paciente, y obliga al médico a dar las explicaciones e informaciones necesarias sobre el diagnóstico y el tratamiento propuesto, así también sobre el pronóstico de su decisión.

Desde la perspectiva del acto médico, es un hecho que no intervenir con medios científicamente comprobados en una patología determinada constituye un acto negligente, pues la obligación del médico es otorgar atención idónea.

El caso del embarazo tubario constituye un dilema moral semejante al triage(12), en el cual siempre se valora toda vida humana, pero, en forma realista, se acepta la imposibilidad de conservar la vida de algunas personas, porque están en situación terminal o por imposibilidad de atención. En estos casos no es que se devalúe algún tipo de calidad de vida o se escoja quién vivirá, sino que la misma razón evalúa una realidad y sus circunstancias.

Ante un embarazo, es obligación que el médico vele por la salud de ambos pacientes, la madre y su hijo, pero en circunstancias que impidan la sobrevida de alguno de ellos, el razonamiento lógico y ético, como es el principio del mal menor, impone salvar la vida del salvable. Ningún médico esta ni ética ni jurídicamente obligado a obtener lo imposible y en este momento esa premisa puede ser aplicada al embrión. En esta patología obstétrica el embrión constituye un paciente terminal, pues independientemente de que se efectúen acciones por prolongar su vida, esta patología acabará en su muerte a corto plazo. En el caso de la madre, es bien sabido que el tiempo de desarrollo de trofoblasto intratubario se encuentra en relación directamente proporcional a la afectación de la salud y posiblemente la muerte materna(13).

Por tanto, tanto la intención del médico como las graves y especiales circunstancias que configuran 
el acto constituyen un objeto que no es un aborto, sino una acción lícita y ordenada éticamente. Es más, en estos casos la omisión de actuar provocaría un mal mayor. Si no se actúa morirían ambos y actuar significa salvar al único posible de salvar, aun si se toman todas las providencias para atender y tratar de salvar al otro.

En general, el primer principio moral que se impone al médico es respetar la vida humana desde su comienzo, proclama incluso -y paradójicamente- de la Declaración de Oslo(14) de la Asociación Médica Mundial sobre el aborto terapéutico. El médico ha de vivir con la máxima sinceridad tal obligación y mostrar un respeto máximo, delicado y atento, tanto a la vida del embrión como a la de la madre. Tal respeto se debe por igual a todo ser humano vivo, independientemente de que su expectativa de vida se cuente en años o en horas o minutos; independientemente de que su edad cronológica se mida en decenios o en días; independientemente de que esa vida transcurra a la luz del día o en el seno de la madre, o de que se desarrolle en la cavidad del útero u ocupe una sede ectópica. Pero la ética debe ser lógica, un embrión que se implanta fuera del útero no tiene posibilidades de crecer ni subsistir; además, supone un riesgo para la vida y la fertilidad de la madre. Ante la evidencia de que el embrión es un ser humano, surge el cuestionamiento de si es éticamente lícito intervenir un ET(15).

De las interrupciones del embarazo antes de la viabilidad fetal por razones de salud materna, solo serían éticamente lícitas las efectuadas cuando el médico tratante está razonablemente convencido de que si no realiza dicha acción sus dos pacientes morirán. En dichos casos no solo existirían circunstancias en las cuales el efecto buscado es proporcional al efecto no deseado, sino que la acción libremente elegida por el médico sería recta, lícita y no constituiría un acto antiético. La acción del médico no será nunca atentar directamente sobre el embrión, sino sobre la causa de la patología, que quizás provoque la muerte del embrión. Un caso semejante es el de la madre con cáncer uterino, que no puede retrasarse en su manejo y que, por razón de la radio o quimioterapia, o por la extirpación directa del tumor, el embrión fallecerá, aunque la intención nunca fue buscar su muerte.

\section{Uso del metotrexate}

El metotrexate(16) (misoprostol) es una droga comúnmente utilizada en oncología, ya que interfiere la síntesis de DNA e inhibe la reproducción celular, efecto deseable en el tejido trofoblástico implantado anormalmente. Ya que el trofoblasto rodea al embrión, éste también recibe el mismo impacto, ocasionando por consecuencia la muerte del mismo $(17,18)$.

La intervención medicamentosa en estos casos ha sido considerada por algunos(19) como una forma de aborto indirecto y, por tanto, en apreciación de la invulnerabilidad de la vida humana, un acto antiético.

Pero la argumentación ética sobre el objetivo de esta intervención puede ser postulada bajo el principio del doble efecto(20). Este principio señala que, para considerar un acto ético, cuando ante una acción médica se presenta un efecto bueno y uno malo, deben cumplirse los siguientes cuatro razonamientos:

El objeto del acto debe ser bueno o por lo menos moralmente neutral. En este caso, el objeto consiste en destruir el trofoblasto, que ocasionará por su crecimiento la ruptura tubaria, lo cual ocasionará la muerte del embrión y provocará una situación crítica o mortal en la madre.

El efecto bueno debe desearse y el malo solamente tolerarse. Lo que se intenta es prevenir la ruptura de la trompa, mas no se desea la muerte del embrión.

El efecto malo no puede ser causa del buen efecto. El mal efecto, muerte del embrión, no es la causa de la cura materna; ya que se evita cuando se impide que el trofoblasto perfore la tuba. Aun en caso de muerte embrionaria, el trofoblasto sigue creciendo, lo que prueba que el trofoblasto es la causa de la patología y no el embrión por sí mismo.

El efecto bueno debe ser proporcional respecto del mal efecto. Prevenir la muerte materna en vez de la muerte de madre e hijo tiene un valor moral, especialmente porque la muerte del embrión no es intencionada. 


\section{Conclusión}

En la actualidad, la ética médica sigue tan vigente como lo fue en la antigüedad, pues es la parte moral del acto médico. Los dilemas morales en el ejercicio de la medicina siempre han acompañado a quienes la ejercen y son cada vez más complejos y delicados en su deliberación y decisión.

Toda persona actúa reflejando su ética y por ello es tan importante la claridad en las decisiones que no son meramente técnicas. El respeto a la conciencia individual está consagrado como un derecho del personal de salud y esto hace que estos temas sean actuales.

Los actos morales, como actos que son, están orientados hacia el exterior, la realidad, el mundo, los demás. Pero, por ser morales, tienen un aspecto interno, que es el que hace que sean valorables. No podemos olvidar que somos morales porque sabemos que podemos elegir, porque sentimos que tenemos posibilidad de seguir caminos diferentes en nuestra vida, porque nos damos cuenta de que nuestras acciones tienen consecuencias. La conciencia de estas consecuencias es la base del aspecto interno de la moral, en ella está el origen de la valoración de nuestros actos, nuestros hábitos o nuestro modo de vida. Pero la conciencia moral es también conciencia de la libertad, conciencia de que no todas las posibilidades de elección son igualmente valiosas. El ser humano libre es aquel que decide responsablemente con base en argumentos adecuados. 


\section{Referencias}

1. Alto WA. Abdominal Pregnancy. New York: AFP; 1990: 41.

2. Cerna RJ. Embarazo ectópico. Ginecología y Obstetricia Aplicadas (Méx.) 2003; 313-322.

3. Ory SJ. Ectopic pregnancy: Current evaluation and treatment. Mayo Clin Proc 1989; 64: 874.

4. Castelazo ME. Embarazo Ectópico triple espontáneo. Ginecol Obstet Mex 2006; 74 (supl): S5-S6.

5. Garrone CM, Broso PR. Tubaric pregnancy: an excellent idea? Med Hypotheses 2000 Jun; 54(6): 900-902.

6. Cobellis L. Use of methotrexate in ectopic pregnancy. Results in 55 patients treated. Minerva Ginecol 1998; 50(12): 513-517.

7. Enríquez Domínguez B, Fuentes González L, Gutiérrez González N, León Barzaga M. Tendencias actuales en el tratamiento del embarazo ectópico. Rev Cubana Obstet Ginecol 2003; 29(3): 43.

8. Barrón VR. Embarazo Ectópico. En: Karchmer KS, Fernández del Castillo SC. Obstetricia y Medicina Perinatal. Temas selectos. Tomo 1, Capítulo 16. México: Colegio Mexicano de Especialistas en Ginecología y Obstetricia; 2006: 165-171.

9. Cerna RJ. Embarazo Ectópico. Ginecologia y Obstetricia Aplicadas (Méx.) 2003; 313-322.

10. Casas Martínez ML. Bases bioéticas para la toma de decisiones en la práctica médica, investigación en bumanos y trasplantes. México: Trillas; 2008: 59.

11. Casas ML, Amor A. Principios Bioéticos y derecho a la salud I. Rev Sanid Milit (Mex.) 2001; 55(1): 27-29.

12. Lähdet EF, Suserud BO, Jonsson A, Lundberg L. Analysis of triage worldwide. Emerg Nurse 2009; 17(4): 16-19.

13. Barnhart KT. Clinical practice. Ectopic pregnancy. N Engl J Med 2009; 361(4): 379-387.

14. World Medical Association. World Medical Association declaration on therapeutic abortion. Disponible en http://www.wma.net/en/30publications/10policies/a1/ Consultado en octubre de 2012.

15. Avilés V. Derecho Obstétrico. Santiago de Chile: Multigráfica S.A.; 1992: 25.

16. Farma Vademecum. Metrotexato. Disponible en http://www.iqb.es/cbasicas/farma/farma04/ m035.htm Consultado en octubre de 2012.

17. Molina Sosa A, Morales Garcia VE. Conservative therapy of ectopic pregnancy with methotrexate. Ginecol Obstet Mex 1993; 61: 201-206.

18. Cobellis L. Use of methotrexate in ectopic pregnancy. Results in 55 patients treated. Minerva Ginecol 1998; 50(12): 513-7.

19. Garcia AJ, Aubert JM, Sama J, Josimovich JB. Expectant management of presumed ectopic pregnancies. Fertil Steril 1987; 48: 395.

20. Sgreccia E. Bioética: manuale per medici e biologi. Milano: Vita e Pensiero; 1986: 164.

Recibido: 13 de abril de 2010

Aceptado: 15 de junio de 2010 